\title{
A Hybrid Convolutional Neural Network and Deep Belief Network for Brain Tumor Detection in MR Images
}

\author{
S.Somasundaram, R.Gobinath
}

\begin{abstract}
Early tumor detection in brain plays vital role in early tumor detection and radiotherapy. MR images are used as the input image for brain tumor finding and classify the type of brain tumor. For early detection or prediction of the brain tumor, an improved feature extraction technique along with Deep Neural Network (DNN) has been recommended. First, MR image is pre-processed, segmented and classified utilizing image processing techniques. Support Vector Machine (SVM) based brain tumor classifications are achieved previously with less precision rate. By integrating DCNN(Deep Convolutional Neural Network) classifier and DBN(Deep Belief Network), an improvement in precision rate can be achieved. This paper mainly focuses on six features viz., entropy, mean, correlation, contrast, energy and homogeneity. The proposed method is used to identify the place, locality and dimension (size) of the tumor in the cerebrum through MR copy using MATLAB software. The performance metrics recall, precision, sensitivity, accuracy and specificity are achieved.
\end{abstract}

Keywords: Brain Tumor; Deep Neural Network; Deep learning NN classifier; Deep Belief Network; Magnetic Resonance Imaging;

\section{INTRODUCTION}

The human cerebrum is perhaps the vital organ which screens all our functional activities that are physical and mental. One such most dangerous deadly cancers in adults is brain tumor. The collection of abnormal cells in some of the tissues in brain referred as brain tumor. Brain tumors of primary and metastatic are the two categories in brain tumors origin. A tumor can be classified as the malignant or benign. A growth without cancer cells is called as benign. They can be removed entirely and it will not reproduce after their removal [1]. The tumor of Benign is less aggressive and it will not spread on nearby tissues. Another type of cancer cell is Malignant, which is more aggressive and it will spread to nearby tissues in brain and additionally in other parts of the body [2]. The main aim is to discover a tumor area in brain at a beginning period. Specialist's take various kinds of pictures of human brain like MRI, PET, and so forth and analyze images to diagnose the tumor. It is exceptionally hard to separate among tumor and different substances seeming like a tumor inside the human brain. So, that brain tumor detection turns into a challenging job [3].

Revised Manuscript Received on July 05, 2019.

S.Somasundaram*, Research Scholar, Department of Computer Science, VISTAS, Chennai, India,

Email: somas2000@gmail.com

Dr.R.Gobinath, Associate Professor, Department of Computer Science, VISTAS, Chennai, India.

Email: iamgobinathmca@gmail.com
The most incessant main brain tumors are gliomas and signify approximately $80 \%$ are malignant brain tumors. Starts from glial cells of the brain or spine and grades are classified as per the cell type. Gliomas grade III and Gliomas grade IV are related with insignificant forecast: patients determined to have glioblastoma multiform make due by and large 12-14 months under treatment [4]. MRI is the sample of medical images is utilized for analysis, treatment arranging and checking of gliomas.

MRI scheme is broadly connected in the field of medical science because of its soft tissue contrast, high spatial resolution, and non-invasive qualities. MRI gives rich data to brain tumor conclusion and treatment arranging. As a brain tumor changes in size, shape and intensity, makes tumor segmentation process progressively repetitive. It gives detailed information symbolisms of the brain, and it is a common amongst the most widely recognized analytic tests estimated for brain tumors [6]. Extraordinary effect for better diagnostics, growth rate treatment and making planning for prediction from MR imageries could be partitioning of brain tumor. It does not produce any dangerous radiation is the main advantage of this technique [7]. This method has an increased contrast of the soft tissues, and serves as a non-invasive scheme. Data is collected with functional structures, and brain tumor partitioning which gives potential irregular tissues on MRI. MRI for brain tumor is shown below fig 1 .

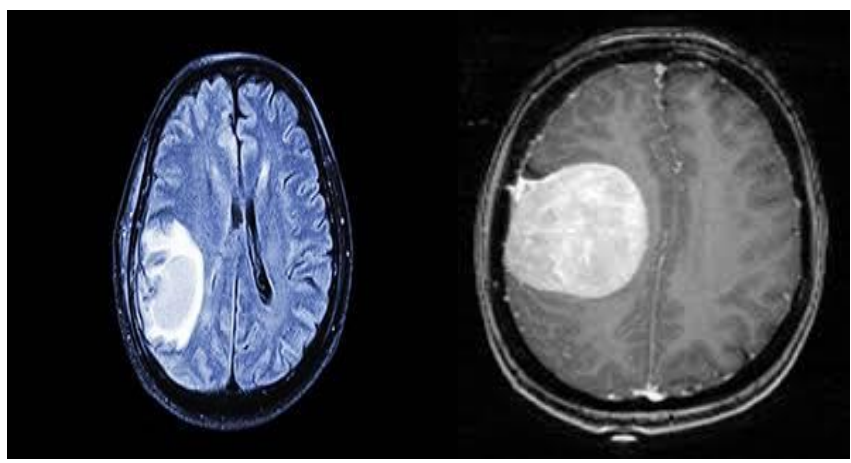

Fig. 1 MRI for brain tumor

In this implementation paper, the image of MRI is used, and it is loaded in mat format. It is changed to grey scale whether it is in RGB format. After the conversion the image will resize and 3 level DWT (Discrete Wavelet Transform) is applied for using median filter. The median filter is utilized to filter the noise in MR image. For reconstruction 2 level IDWT is applied in the image. The fast fuzzy segmentation is utilized for segment the tumor and then segmented image is processed in the Convolutional Neural Network (CNN). Using CNN,

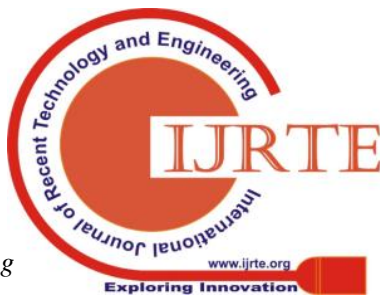


the feature is extracted by convolution process. These features are converted into binary form because Deep Belief Network is used in the process of classification. Using this method 3 types of tumors can be classified as meningioma, gliomas \& pituitary tumor.

\section{RELATED WORK}

Mlynarski et al. (2019) introduced a CNN classifier model that effectively combined the benefits of the long-range two-dimensional context and the short-range three-dimensional context. An architecture model was proposed in which the distinct sequences of MR are processed by individual subnetworks for producing a model that is strong to the issue of lost MR sequences. This technique can be naturally employed on several tasks relating to the segmentation of organs or lesions. The limitation of the study was that it ignored the mass effect produced by the tumor and it might lead to unsuitable registration [1].

Anaraki et al. (2019) proposed the structure of the Convolution Neural Network which was developed with GA, different existing techniques of choosing a DNN that were generally founded on trial and error method or by predefined normal structures. Moreover, to minimize the change of bagging, prediction error as an collaborative algorithm was used on finest model developed by the GA. Because of flexible nature of the technique, it can be promptly utilized practically for helping the medic to analyze brain tumors at initial stage. This method still wants to give better performance to improve the accuracy [4].

Yu et al. (2018) presented a novel segmentation technique known as Crossbar-Net, with the objective of precise segmentation of the kidney tumors. They presented a flowed training technique that successfully guaranteed the uniformity between sub models, by feeding each other with the complex samples, to get better segmentation. The way of symmetric data from parallel and vertical to other axis is the main drawback [6].

Amin et al. (2018) used 07 layers for classification which include 03 ReLU, a softmax layer, and 03 convolutional layers. The input image of MR image was separated into various patches and the middle value of the pixels was supplied to DNN. DNN assigns labels with reference to the pixel at the center and performs the process of segmentation. The results were evaluated based on sensitivity (SE), accuracy (ACC), specificity (SP), precision, Dice Similarity Coefficient (DSC), Jaccard similarity index (JSI), TPR(True Positive Rate) and FPR(False Positive Rate) [7].

Bernal et al. (2018) presented a broad literature study of CNN methods applied in brain MRI study, concentrating on the designs, pre-processing, data preparation and post-processing procedures that were existing in this work. The main motive of the study was 3 -fold. The main objective was to report how various CNN structures were developed, discussed about advanced techniques, concise their outcomes achieved with the datasets and discussed their disadvantages as well as advantages. However, because of the absence of labelled training input and limitations of computational power, it is not viable to train deep Convolution Neural Networks without over-fitting [8].

Cui et al. (2018) presented a novel completely automated technique of segmentation in vivo brain gliomas from MRI dataset. This methodology failed to locate the entire region of the tumor and could precisely isolate the intra-tumor structure. This work depended on a cascaded type Convolution Neural Network comprising of two sub-networks: TLN(Tumor Localization Network) and next was an ITCN(Intra-Tumor Classification Network). As an extension of DNN, histological information and other information can be included to increase the clinical management of brain tumor [10].

Saouli et al. (2018) proposed three end-to-end Incremental DCNN models for completely automated segmentation of the brain tumor. These methods were found unlikely compared as compared to other Convolution Neural Networks based models that follow the strategy of trial and error process which didn't utilize any directed approach to deal with the reasonable hyper-parameters. For resolving of the issue of training CNN models, a training technique which considers the impacting hyper-parameters by bounding and fixing a roof to these hyper-parameters to speed-up the training was advised[12].

Sajjad et al. (2018) proposed a CNN based classification of multiple grade brain tumors. At first, tumor areas were segmented by utilizing a deep learning method. Furthermore, broad data augmentation was utilized to successfully train the framework, evading the absence of data issue when dealing with MR images for multiple grade brain tumor classification. Finally, a pre-trained Convolution Neural Network classifier was tuned with expanded information for the classification of brain tumors. Developing the work for the classification of fine-grained with study of CNN structure was a complicated process and cannot be developed easily [14].

Wang et al. (2018) suggested a new deep learning interactive framework of segmentation by integrating CNN models into a bounding box and scribble type pipeline of segmentation. An image-specific modified tuning was suggested for making a CNN framework to work adaptively to the specified test image, that could be done with supervised or unsupervised. A weighted loss function considered in the network along with uncertainty on the basis of interaction for the fine tuning was also proposed in this study [15].

Takács et al. (2018) proposed a brain tumor segmentation technique dependent on visual saliency features on MR image volumes. This technique utilized a novel integration of several MRI modalities in addition to highlight the potential tumors by applying a healthy template generated from the slices of annotated database in the absence of tumors. This approach was likewise merged with $\mathrm{CNN}$ to minimize the networks eventual over-fitting which could gave weaker predictions for concealed cases. However, it will not handle pictures with varying protocols from ones utilized for obtaining the training information [16].

\section{III.PROPOSED METHODOLOGY}

The suggested method for brain tumor detection and classification in MRI is separated into 4 steps. The flow diagram of the advised methodology is given in the Fig. 2. Initially, MRI images are loaded as input images. MR images are given as input to the pre-processing block. The image file is loaded as mat format and it is converted to gray scale image. In this stage, images are pre-processed which means that the size and shape of the images are improved. The size of the MRI image will be resized into $512 * 512$ 
pixels. In this pre-processing, three-level DWT (Discrete Wavelet Transform) is applied for using median filter. Then the median filter is used to filter the noise in MR images.

After that three-level IDWT (Inverse Discrete Wavelet Transform) is applied for reconstruct the image. The output of the pre-processing stage images is given as input to the image segmentation block. In this stage, segmentation is done using the fast fuzzy segmentation which is used for segment the tumor in the image. The outputs of the image segmentation are given as input to the classification. In the classification stage, the images are classified by using CNN (Convolutional Neural Network) with the combination of DBN (Deep Belief Network) for better improvement in accuracy. In CNN, we extract the feature by convolution process. The extracted features are converted into binary form because for using DBN.

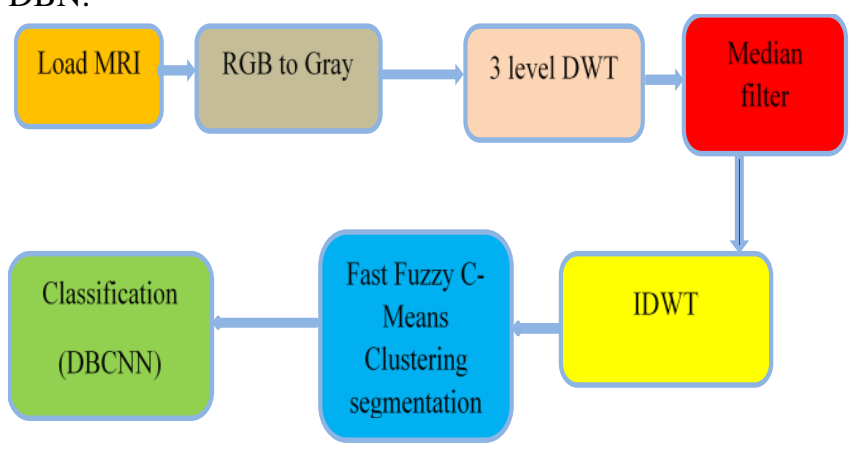

Fig.1. Flow Diagram of Proposed Methodology

After feature extraction classification process is done, different classification techniques are used such as an artificial neural network, DNN, convolutional neural network, etc. like. After classification, the simulation results are validated. Our suggested methodology is based on the Deep Belief Convolutional Neural Network (DBCNN) learning architecture for classification where the classifier is used to identify brain tumors in MR Images.

\section{A. PRE-PROCESSING}

The image is loaded as the .mat format and the image is in the form of MRI. MRI image is in either RGB or gray scale. The image is resized and converted to grey if the image was in color. After that DWT (Discrete Wavelet Transform) will be applied.

\section{i. Discrete Wavelet Transform}

DWT is used for edge detection, de-correlation, time-frequency localization and multi-resolution study had utilized in MR image de-noising. It separate noise by separating coefficients that were not important with respect to some threshold. The choice of this threshold chooses the acceptability of de-noising [21]. DWT is a technique that gathers the data about the time and frequency for that only DWT is preferred. It is making better by maximizing the levels of DWT that is 2-level DWT, 3-level DWT and so on. DWT transform method divide the image into four parts and the properties of human visual system precisely reflected by the coefficients [22]. Apply 3- level DWT in gray scale image and its separate the image for applying median filter.
Median filter is a pre-processing step for improving results and it is mainly used for removing noise and smoothing image regions. It is an edge-preserving and non-linear de-noising smoothing filter for the input images. In this median filter, the selected noisy pixels are changed by median values of their neighbors. The filtering window size decides the number of neighbors. The mid element in a classified sequence is described as the median value [27].

$\operatorname{Median}(\mathrm{A})=\operatorname{Med}\{\mathrm{Ai}\}$

$=\operatorname{Ai}(\mathrm{t}+1) / 2, \mathrm{t}$ is odd

$=1 / 2[\operatorname{Ai}(\mathrm{t} / 2)+\operatorname{Ai}(\mathrm{t} / 2)+1], \mathrm{t}$

\section{iii. Inverse discrete wavelet transform}

Inverse discrete wavelet transform (IDWT) of input or reconstruction signals from the sub channel bands are done using slower rates of samples and smaller bandwidths.

$$
\begin{aligned}
& 1(2 c, d)=11(c, d)-\beta(\operatorname{lh}(\mathrm{c}, \mathrm{d})+\mathrm{lh}(\mathrm{c}-1, \mathrm{~d})) \\
& 1(2 \mathrm{c}+1, \mathrm{~d})=\mathrm{lh}(\mathrm{c}, \mathrm{d})-\alpha(\mathrm{L}(2 \mathrm{c}, \mathrm{d})+\mathrm{l}(2 \mathrm{c}+2, \mathrm{~d})) \\
& (2 \mathrm{c}, \mathrm{d})=\mathrm{hl}(\mathrm{c}, \mathrm{d})-\beta(\mathrm{hh}(\mathrm{c}, \mathrm{d})+\mathrm{hh}(\mathrm{c}-1, \mathrm{~d})) \\
& \mathrm{h}(2 \mathrm{c}+1, \mathrm{~d})=\mathrm{hh}(\mathrm{c}, \mathrm{d})-\alpha(\mathrm{h}(2 \mathrm{c}, \mathrm{d})+\mathrm{h}(2 \mathrm{c}+2, \mathrm{~d})) \\
& \mathrm{x}(\mathrm{c}, 2 \mathrm{~d})=1(\mathrm{c}, \mathrm{d})-\beta(\mathrm{h}(\mathrm{c}, \mathrm{d})+\mathrm{h}(\mathrm{c}, \mathrm{d}-1)) \\
& \mathrm{x}(\mathrm{c}, 2 \mathrm{~d}+1)=\mathrm{h}(\mathrm{c}, \mathrm{d})-\alpha(\mathrm{x}(\mathrm{c}, 2 \mathrm{~d})+\mathrm{x}(\mathrm{c}, 2 \mathrm{~d}+2))
\end{aligned}
$$

\section{B. IMAGE SEGMENTATION}

Medical image segmentation is a labelling process for each pixel in a medical image data set for indicating the type of tissue or the structure of anatomy. The labels that result from this approach have a vast area of applications in visualization and medical research. The method of dividing an image into sets of pixels which is also called super pixels is the image segmentation. The chief objective of segmentation is to identify the tumor's location. It is along chosen by the pixel powers themselves, yet in addition by the neighboring pixel powers and locations. Thought of these neighboring pixels extraordinarily controls the impact of noise [24].

\section{i. Fast Fuzzy C-Means Clustering}

In the proposed method, the fast Fuzzy C-Means Clustering (FCM) algorithm is used for segmentation. The difference between the pixel intensity and center of cluster is the count of FCM and it is non-resistive. It is mainly depending on an objective function relating to fuzzy membership and set of cluster centroids. The popular technique for maximizing sturdiness of FCM of noise to alter the objective function. The information component with more than one cluster and connected with each component is a lot of membership levels. This paper is view of investigating the execution of FCM algorithm. It is an improvement of prior clustering algorithms. Fuzzy clustering is a procedure of allocating enrollment level to each information component and afterward allocates the information component to more clusters. It depends on reducing the function, with regard to enrollment value and cluster focuses [25]

$$
F(x, y)=\sum_{j=1}^{W} \sum_{k=1}^{e}\left(\mu_{j k}\right)^{m}\left\|u_{j}-v_{k}\right\|^{2}
$$

Where,

$\mathrm{N}=$ number of data points, $v_{k}=k^{\text {th }}$ cluster center, $\mathrm{m}=$ fuzziness index $\mathrm{m}, \mathrm{c}=$ the number of cluster center, $\mu_{i k}=$

\section{ii. Median filter}


membership of $j^{\text {th }}$ data to $k^{\text {th }}$ cluster center

$\mu_{j k}=\frac{1}{\sum_{k=1}^{c} \frac{\| w_{j}-v_{j j}}{\left\|w_{j}-v_{k k}\right\|} \mid \frac{2}{m-1}}$

$\left\|u_{i}-v_{j}\right\|=$ Euclidean distance between $j^{\text {th }}$ data and $k^{\text {th }}$ cluster center.

\section{CLASSIFICATION - DBCNN}

Increase of precision and more information from each class is obtained in the classification. These results are dependent on the number of classes choose by the user. To get good classification quality, it is important to select an exact number of classes K. In the presented method DBCNN with the mixture of DBN is used for classification of the image. CNN's use a variation of multilayer perceptron's designed for the need of minimal preprocessing. Two types of CNN's Pooling, Convolutional, and using DBN for classification. The existing layer's feature maps are involved with knowledgeable kernels and put along the activation function to establish the results of the feature map at a convolutional layer. Every resultant map may merge convolutions with various input maps.

The kernels weights are adjusted throughout the training stage, so as to increase the qualities of the information by back propagation. Convolutional layers have less weights to prepare thick FC layers, making CNN simpler to train and less inclined to over fitting, since the kernels are shared among all units of a similar component maps [26].

After classification DBN will be used for identify the brain tumor. It is an automatic classification method and it is mainly focus on a DBN is a productive graphical demonstration, or even a system of neurons deep in machine learning type, comprise of a many layers of inactive factors, connections inside the layers, yet not inside the units, between each layer. A DBN can figure out how to probabilistically recreate its input at the point when prepared on a lot of examples in an unsupervised way. The layers at that point act as feature identifiers on data sources. After this learning, it performs classification, a DBN can be further prepared supervised [26]. DBNs will be considered as a straightforward arrangement of unsupervised system, for example, Restricted Boltzmann Machines (RBMs), in which each sub layer hidden network fills in as the obvious layer for the following. This prompts a speedy, layer by layer, the unsupervised preparing strategy where the contrastive difference is connected to each subnet, beginning with the 'down' pair of layers. While preparing on a lot of tests in an unsupervised way DBN can ready to learn and probabilistically it could remake its inputs. And also for observation and back propagation neural systems, DBN serves as an unsupervised learning technique. A conviction belief network is created stochastic of paired units with weighted connections [27].

\section{IV.RESULTS AND DISCUSSION}

For performance evaluation of the present method, the early stage brain tumor identification is done. The resulting images are compared to the original images, and the location of a tumor in the brain is found. Resultant images of image pre-processing, image segmentation and classification are mentioned below. Input MR image is resized into $512 * 512$. Then the resized image is converted into the grey scale image if the MRI image is RGB color. The input image is shown in fig. 2

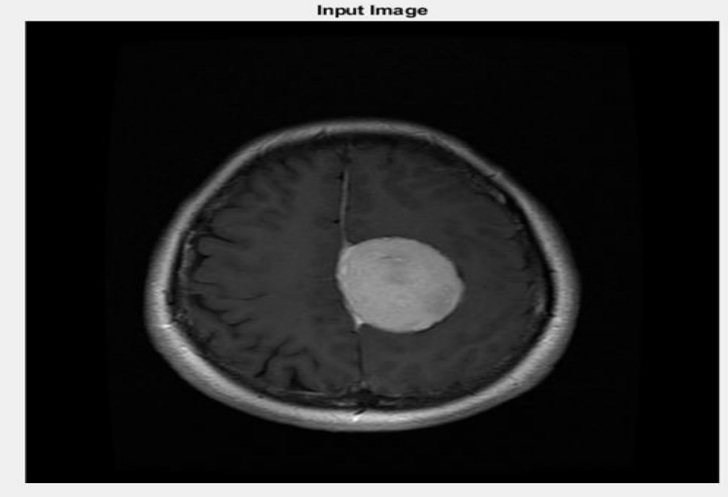

Fig.2 Input image of brain tumor

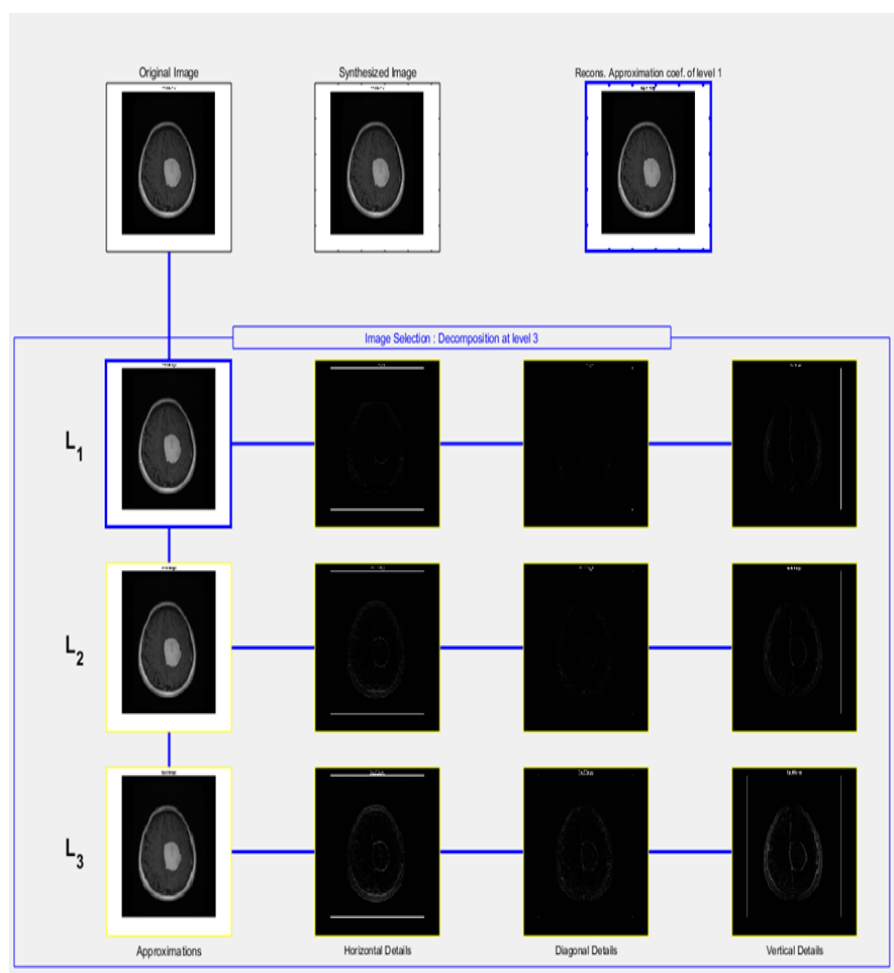

Fig 3: 3-level DWT

Median filter is applied in 3- level DWT. It is applied in each separated image and remove the unwanted noise and it makes the image fine to identify the tumor in brain. By applying the median filter, noise is removed the noise as shown in fig 4 .

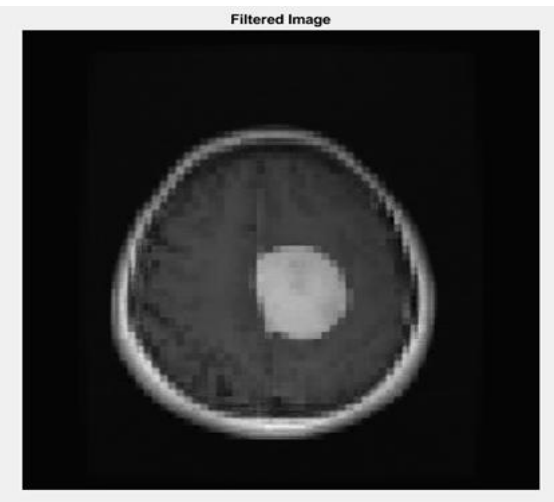

Fig 4 filtered image

The filtered image is partitioned using the fast fuzzy c-means 
clustering algorithm. In FCM technique threshold value is applied in the brain image. In the segmentation process, the brain tumor is extracted from the filtered image as shown in fig 5 .

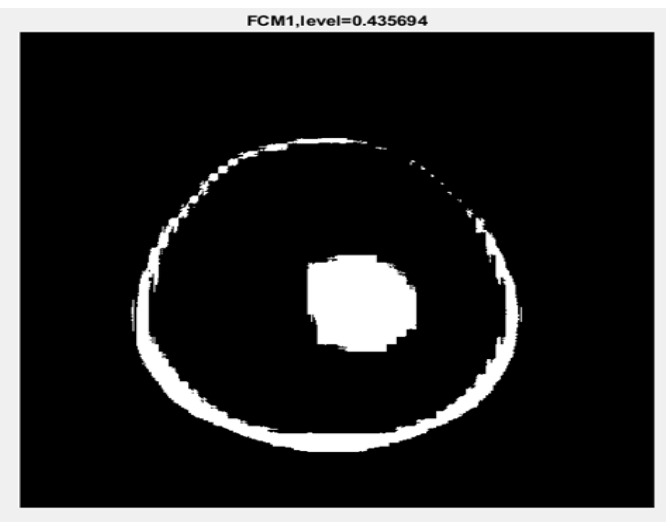

Fig.5 Segmented Image

Segmented images are used as the input to the CNN and DBN for the classification process. The mixture of $\mathrm{CNN}$ and DBN classification will identify the tumor and it will classify which type of tumor. It gives result of classified brain tumor and it will identify it.

Performance evaluation of the proposed methodology is done by calculating three performance metrics namely, accuracy, sensitivity and specificity. Calculations are carried out with the help of a confusion matrix which contains TP (True Positive(TP), TN(True Negative), False Negative (FN), and False Positive (FP) values. The confusion matrix of the suggested DBCNN classifier is shown in the Fig.6.

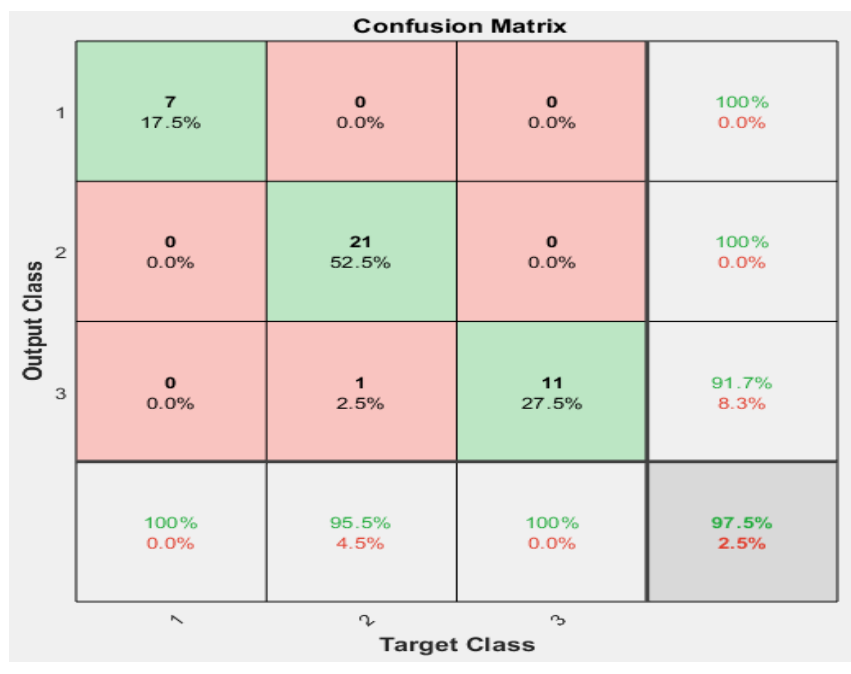

Fig. 6. Confusion matrix of DBCNN

Accuracy: The quantity of forecast in the absence of taking into description which one is positive and which one is negative is the accuracy. Accuracy calculated as below.

accuracy $=\frac{T P+T N}{T P+T N+F P+F N}$

Sensitivity: The proportion of correct positives, the capacity of the network to exactly forecast the form for occurrence that literally has it is the sensitivity. The sensitivity is calculated with the below equation.

sensitivity $=\frac{T P}{T P+F N}$

Specificity: The proportion of correct negatives, the network's capacity to exactly predict without the condition for occurrence that doesn't have it exactly. The specificity calculation is done by the below equation

specificity $=\frac{T N}{T N+F P}$

Accuracy, sensitivity, specificity for the existing PRNN model [18] are compared with the performance metrics of the proposed DBCNN classifier for validation. The comparative results are tabulated in the Table 1.

Table 1 Comparison results of PRNN method and proposed DBCNN method

\begin{tabular}{|c|c|c|c|c|c|}
\hline Classifier & Accuracy & Sensitivity & Specificity & Precision & Recall \\
\hline KNN & $59.86 \%$ & $40 \%$ & $90 \%$ & $77.68 \%$ & $64.98 \%$ \\
\hline PRNN [18] & $74.35 \%$ & $86.93 \%$ & $49.18 \%$ & $78.23 \%$ & $65.08 \%$ \\
\hline Proposed DBCNN & $97.5 \%$ & $100 \%$ & $100 \%$ & $94.20 \%$ & $99.57 \%$ \\
\hline
\end{tabular}

Above comparative results show that the proposed DBCNN classifier is superior in accuracy, sensitivity and specificity compared to the implemented metrics of PRNN.

\section{CONCLUSION AND FUTURE WORK}

The methodology of early tumor detection in the brain plays a important part in premature tumor detection and radiotherapy planning. In this study, MRI images are used as the input for brain tumor identification and categorization. An early brain tumor identification and classification is proposed based on the DNN. Initially, the MRI image will be pre-processed, segmented and feature extracted using the techniques that are included in image processing and then classified utilizing deep learning NN classifier. The same process is repeated for 50 images, and the results are found to be efficient. The performance metrics like precision, recall, and accuracy are calculated, and is proved that the proposed DBCNN classifier is better compared to existing PRNN classifier. The precision rate is improved for the DBCNN classifier. Thus the proposed work can further be used in the compression technique of image processing. Future work can be continued with four level DWT and five level DWT. 


\section{REFERENCES}

1. Mlynarski, P., Delingette, H., Criminisi, A., \& Ayache, N. (2019). 3d convolutional neural networks for tumor segmentation using long-range $2 \mathrm{~d}$ context. Computerized Medical Imaging and Graphics.

2. Chen, S., Ding, C., \& Liu, M. (2019). Dual-force convolutional neural networks for accurate brain tumor segmentation. Pattern Recognition, 88, 90-100.

3. Diniz, J. O. B., Diniz, P. H. B., Valente, T. L. A., Silva, A. C., \& Paiva, A. C. (2019). Spinal cord detection in planning $\mathrm{CT}$ for radiotherapy through adaptive template matching, IMSLIC and convolutional neural networks. Computer methods and programs in biomedicine.

4. Anaraki, A. K., Ayati, M., \& Kazemi, F. (2019). Magnetic resonance imaging-based brain tumor grades classification and grading via convolutional neural networks and genetic algorithms. Biocybernetics and Biomedical Engineering, 39(1), 63-74.

5. Wang, C., Tyagi, N., Rimner, A., Hu, Y. C., Veeraraghavan, H., Li, G., ... \& Zhang, P. (2019). Segmenting lung tumors on longitudinal imaging studies via a patient-specific adaptive convolutional neural network. Radiotherapy and Oncology, 131, 101-107.

6. Yu, Q., Shi, Y., Sun, J., Gao, Y., Dai, Y., \& Zhu, J. (2018). Crossbar-Net: A Novel Convolutional Network for Kidney Tumor Segmentation in CT Images. arXiv preprint arXiv:1804.10484.

7. Amin, J., Sharif, M., Yasmin, M., \& Fernandes, S. L. (2018). Big data analysis for brain tumor detection: Deep convolutional neural networks. Future Generation Computer Systems, 87, 290-297.

8. Bernal, J., Kushibar, K., Asfaw, D. S., Valverde, S., Oliver, A., Martí, R., \& Lladó, X. (2018). Deep convolutional neural networks for brain image analysis on magnetic resonance imaging: a review. Artificial intelligence in medicine.

9. Charron, O., Lallement, A., Jarnet, D., Noblet, V., Clavier, J. B., \& Meyer, P. (2018). Automatic detection and segmentation of brain metastases on multimodal MR images with a deep convolutional neural network. Computers in biology and medicine, 95, 43-54.

10. Cui, S., Mao, L., Jiang, J., Liu, C., \& Xiong, S. (2018). Automatic semantic segmentation of brain gliomas from MRI images using a deep cascaded neural network. Journal of healthcare engineering, 2018.

11. Hasan, S. K., \& Linte, C. A. (2018, October). A Modified U-Net Convolutional Network Featuring a Nearest-neighbor Re-sampling-based Elastic-Transformation for Brain Tissue Characterization and Segmentation. In 2018 IEEE Western New York Image and Signal Processing Workshop (WNYISPW) (pp. 1-5). IEEE.

12. Saouli, R., Akil, M., \& Kachouri, R. (2018). Fully automatic brain tumor segmentation using end-to-end incremental deep neural networks in MRI images. Computer methods and programs in biomedicine, 166, 39-49.

13. Rezaei, M., Yang, H., \& Meinel, C. (2018, July). Instance tumor segmentation using multitask convolutional neural network. In 2018 International Joint Conference on Neural Networks (IJCNN) (pp. 1-8). IEEE.

14. Sajjad, M., Khan, S., Muhammad, K., Wu, W., Ullah, A., \& Baik, S. W. (2019). Multi-grade brain tumor classification using deep $\mathrm{CNN}$ with extensive data augmentation. Journal of computational science, 30, 174-182.

15. Wang, G., Li, W., Zuluaga, M. A., Pratt, R., Patel, P. A., Aertsen, M., ... \& Vercauteren, T. (2018). Interactive medical image segmentation using deep learning with image-specific fine tuning. IEEE transactions on medical imaging, 37(7), 1562-1573.

16. Takács, P., \& Manno-Kovacs, A. (2018, September). MRI Brain Tumor Segmentation Combining Saliency and Convolutional Network Features. In 2018 International Conference on Content-Based Multimedia Indexing (CBMI) (pp. 1-6). IEEE.

17. Hussain, S., Anwar, S. M., \& Majid, M. (2017, July). Brain tumor segmentation using cascaded deep convolutional neural network. In 2017 39th Annual International Conference of the IEEE Engineering in Medicine and Biology Society (EMBC) (pp. 1998-2001). IEEE.

18. Mohsen, H., El-Dahshan, E. S. A., El-Horbaty, E. S. M., \& Salem, A. B. M. (2018). Classification using deep learning neural networks for brain tumors. Future Computing and Informatics Journal, 3(1), 68-71.

19. Chato, L., \& Latifi, S. (2017, October). Machine learning and deep learning techniques to predict overall survival of brain tumor patients using MRI images. In 2017 IEEE 17th International Conference on Bioinformatics and Bioengineering (BIBE) (pp. 9-14). IEEE.

20. Mengqiao, W., Jie, Y., Yilei, C., \& Hao, W. (2017, September). The multimodal brain tumor image segmentation based on convolutional neural networks. In 2017 2nd IEEE International Conference on Computational Intelligence and Applications (ICCIA) (pp. 336-339). IEEE.

21. Aravindan, T. E., Seshasayanan, R., \& Vishvaksenan, K. S. (2018). Medical image denoising by using discrete wavelet transform: Neutrosophic theory new direction. Cognitive Systems Research.

22. Choudhary, R., \& Parmar, G. (2016, November). A robust image watermarking technique using 2-level discrete wavelet transform (DWT). In 2016 2nd International Conference on Communication Control and Intelligent Systems (CCIS) (pp. 120-124). IEEE. 
23. George, G., Oommen, R. M., Shelly, S., Philipose, S. S., \& Varghese, A. M. (2018, March). A Survey on Various Median Filtering Techniques For Removal of Impulse Noise From Digital Image. In 2018 Conference on Emerging Devices and Smart Systems (ICEDSS) (pp. 235-238). IEEE.

24. Shen, S., Sandham, W., Granat, M., \& Sterr, A. (2005). MRI fuzzy segmentation of brain tissue using neighborhood attraction with neural-network optimization. IEEE transactions on information technology in biomedicine, 9(3), 459-467.

25. Preetha, R., \& Suresh, G. R. (2014, February). Performance analysis of fuzzy c means algorithm in automated detection of brain tumor. In 2014 World Congress on Computing and Communication Technologies (pp. 30-33). IEEE.

26. Thahseen, P., \& Anish, K. B. (2017, July). A Deep Belief Network Based Brain Tumor Detection in MRI Images. International Journal of Science and Research (IJSR), Volume 6 Issue 7.

27. Anishya, F., Giridharan, S., \& Hemalatha, P. (2018). Brain Tumor Prognosis and Prediction using Deep Belief Network. International Journal of Pure and Applied Mathematics, (pp-3087-3096).

28. Rohilla, M., \& Singal, V. (2014). Design and Comparative Analysis of SVM and PR Neural Network for Classification of Brain Tumour in MRI. International Journal of Computer Applications, 91(17).

\section{AUTHORS PROFILE}

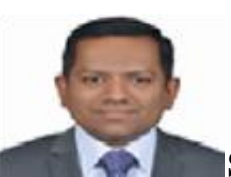

S.Somasundaram completed Bachelor of Physics in Madras University and Master of Computer Application in Bharathiyar university at Coimbatore, India. Currently working in reputed IT company and have 20+ years of experience in IT Industry. Worked in several countries US, Australia and Europe. Currently pursuing Doctoral degree in Vels Institute of Science, Technology and Advanced studies (VISTAS), Chennai, India. Interests are Digital Image Processing and Neural Network.

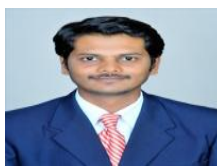

Dr. R.Gobinath is a Ph.D holder from Karpagam University, Coimbatore and Master of Computer Application from Anna University, India. Currently working as a Associate Professor in Vels Institute of Science, Technology and Advanced Studies (VISTAS), Chennai, India. He has 21 International Journal publications and 16 conference publications. His interests are Web mining, Image Processing and Pattern recognition. 